\title{
利用鸡胚模型半体内评价多孔磷酸钙骨修复 材料血管化的研究
}

\author{
肖 雯 ${ }^{1}$, 刘玉梅 ${ }^{1}$, 任凯歌 ${ }^{2}$, 匙 峰 ${ }^{1}$, 李 焰 ${ }^{2}$, 智 伟 $^{1}$, 翁 杰 $^{1}$, 屈树新 ${ }^{1}$
}

(1. 西南交通大学 材料先进技术教育部重点实验室, 材料科学与工程学院, 成都 $610031 ; 2$. 成都军区总医院口腔 科, 成都 610083)

摘 要: 利用鸡胚线毛尿囊膜(CAM)中丰富的血管建立半体内模型，研究多孔磷酸钻骨修复材料的血管化。将多孔 羟基磷灰石陶瓷(HA)和具有部分孔隙结构的磷酸锠骨水泥(CPCs)分别植入 CAM 中共培养，利用体式显微镜和扫 描电子显微镜(SEM)观察 CPCs 表面血管的生长, 并利用 Image-Pro Plus 和 Nano Measurer 定量血管密度、直径和数 量; 对 CAM 中培养的 HA 采用不脱钻组织学处理和观察。体式显微镜观察及定量结果显示多孔结构对血管生长 影响显著，随着培养时间延长，多孔部分血管密度和数量均有明显增加，而无孔部分血管生长缓慢。CPCs 表面 血管以出芽方式生长, 形成血管网络, 直径一般小于 $50 \mu \mathrm{m}$ 。SEM 观察显示血管紧贴 CPCs 表面生长。苏木精-伊 红(H\&E)染色显示血管通过孔隙结构长入 HA 内部。实验结果说明三维孔隙结构利于血管的生长和长入，此实验可 简便、高效、低成本地研究多孔磷酸钘骨修复材料的血管化，为快速评价生物材料血管化寻找一种新途径。

关 键 词: 鸡胚线毛尿囊膜; 多孔磷酸钲骨修复材料; 血管化; 半体内

中图分类号: TB321 文献标识码: A

\section{Evaluation of Vascularization of Porous Calcium Phosphate by Chick Chorioallantoic Membrane Model ex vivo}

\author{
XIAO Wen ${ }^{1}$, LIU Yu-Mei ${ }^{1}$, REN Kai-Ge ${ }^{2}$, SHI Feng ${ }^{1}$, LI Yan $^{2}$, ZHI Wei ${ }^{1}$, WENG Jie ${ }^{1}$, QU Shu-Xin ${ }^{1}$ \\ (1. Key Lab of Advanced Technologies of Materials, Ministry of Education, School of Material Science and Engineering, \\ Southwest Jiaotong University, Chengdu 610031, China; 2. Department of Stomatology, Chengdu Army General Hospital, \\ Chengdu 610083, China)
}

\begin{abstract}
In this study, ex vivo chick chorioallantoic membrane (CAM) model with abundant blood vessels was developed to investigate the vascularization of porous calcium phosphate. Porous hydroxyapatite ceramic (HA) and calcium phosphate cement with segmental porous structure (CPCs) were implanted into CAM, respectively. Stereomicroscope and scanning electron microscope (SEM) were employed to observe the angiogenesis on the surface of CPCs. The vascular density, diameters and numbers were quantified by Image-Pro Plus and Nano Measurer. Furthermore, undecalcified histological staining was conducted to observe the inner angiogenesis of porous HA. The vascular den-
\end{abstract}

收稿日期: 2016-09-22; 收到修改稿日期：2016-11-02

基金项目: 国家重点基础研究发展计划(973)项目(2012CB933602); 国家自然科学基金(51372210); 四川省应用基础(重点)项目 (2016JY0011); 教育部博士点基金(20130184110023); 中央高校基本科研业务费专项基金(2682016YXZT11); 四川省 高校科研创新团队建设计划项目(14TD0050)

National Basic Research Program of China (973 Program, 2012CB933602); National Natural Science Foundation of China (51372210); Basic Research Foundation Key Project of Sichuan Province (2016JY0011); Research Fund for the Doctoral Program of Higher Education of China (20130184110023); Fundamental Research Funds for the Central Universities (2682016YXZT11); The Construction Program for Innovative Research Team of University in Sichuan Province (14TD0050)

作者简介：肖 雯(1993-)，女，硕士研究生. E-mail: 18728428596@163.com

通讯作者: 屈树新, 教授. E-mail: qushuxin@swjtu.edu.cn 
sity and number in the porous part of CPCs both increased significantly with the increase of culture time, while the angiogenesis was tardily in the part of CPCs without pores. Most of the blood vessels less than $50 \mu \mathrm{m}$ on the porous part of CPCs grew in budding manner to form vascular network. SEM images showed that blood vessels grew tightly on the surface of CPCs. Histological staining demonstrated that some blood vessels grew into the inside of porous HA. These results suggest that three-dimensional porous structure may promote the angiogenesis of porous calcium phosphate and the present CAM model may provide a convenient, efficient and with low-cost approach to evaluate vascularization of porous biomaterials.

Key words: chick chorioallantoic membrane; porous calcium phosphate materials; vascularization; ex vivo

多孔磷酸钙骨修复材料不仅具有与人体骨组织 相似的无机成分，而且具有仿骨组织的三维孔隙结构, 因此具有良好的生物相容性和骨传导性等, 已成功应 用于临床。其中仿生三维孔隙结构适于细胞和组织的 长入, 利于材料的血管化, 促进新骨的形成 ${ }^{[1]}$ 。因此, 骨修复材料血管化是其成功应用于临床的关键。目前, 主要采用动物植入实验, 结合组织学和血管造影技术 等研究多孔磷酸钻骨修复材料的血管化 ${ }^{[2-4]}$ 。但动物实 验的操作和影响因素复杂、研究周期长、成本较高, 且 难以直接观察血管长入材料的动态变化。

鸡胚线毛尿囊膜(Chick chorioallantoic membrane, $\mathrm{CAM}$ ) 是鸡胚发育到第 4 5 d, 尿囊膜中胚层外层与线 毛膜中胚层内层融合而形成的, 随着鸡胚的发育, 膜 中血管网络逐渐形成, 孵育约至第 $8 \mathrm{~d}$ 后, 血管网络 发育趋于稳定 ${ }^{[5]}$ 。CAM 中丰富的血管组织是研究活体 血管形成与抗血管形成理想的半体内模型。相比动物 实验, CAM 模型操作简便、实验周期短、成本低廉、 易于样本大量重复并且便于直接观察血管动态生长 过程。已有研究利用 CAM 模型进行与血管生成相关 的研究, 并定性或定量评价, 如应用于肿瘤模型的建 立 $^{[6]}$ 、肿瘤治疗的优化 ${ }^{[7-8]}$ 、血管相关疾病机理的研究 ${ }^{[9]}$ 和血管相关药物药效的评价 ${ }^{[10]}$ 。最近有研究利用 CAM 模型研究骨修复材料的生物相容性 ${ }^{[11]}$, Magnaudeix 等 ${ }^{[12]}$ 利用 CAM 模型定量评价具有不同孔隙 形状的含 $\mathrm{Si}$ 差基磷灰石支架对血管生长的影响, 但由 于长入多孔材料的组织有限以及磷酸钙骨修复材料 力学性能的限制, 很难利用常规的组织学技术评价骨 组织修复材料孔隙中的血管化。此外, 现有研究仅在 固定时间观察材料表面血管生长的情况 ${ }^{[13]}$ 。目前尚未 见关于多孔磷酸钙骨修复材料孔隙中血管动态生长 及利用组织学技术评价血管长入的研究。

本研究拟利用 CAM 模型评价多孔磷酸钻骨修复 材料的血管化。采用体式显微镜动态观察、软件定量 分析多孔磷酸钙骨水泥(Calcium phosphate cement, $\mathrm{CPC}$ )表面的血管生长, 比较 $\mathrm{CPC}$ 中多孔和无孔结构
对血管生长的影响, 利用扫描电子显微镜观察 $\mathrm{CPC}$ 表 面血管生长情况。由于植入 $\mathrm{CAM}$ 后培养时间较短, 长 入多孔 CPC 内部的血管和组织较少, 脱钙后材料/组 织不能保持完整结构, 同时 $\mathrm{CPC}$ 力学强度较差, 不脱 钙切片时, 易碎成粉末, 难以进行组织学分析, 其制 片方法正在进一步研究。而烧结后的多孔羟基磷灰石 陶瓷(Hydroxyapatite ceramic, HA)力学强度增强, 在 组织长入有限的情况下可制备不脱钙组织学切片。因 此本实验选用多孔 HA 进行血管化的组织学评价, 研 究血管在多孔磷酸钙材料内部的生长情况。本工作通 过磷酸钙骨水泥表面血管生长和多孔羟基磷灰石内 部血管生长的评价, 研究了三维孔隙结构对多孔磷酸 钙骨修复材料血管化的影响; 为简便、快速、定量评 价生物材料血管化提供一种参考方法。

\section{1 实验方法}

\section{1 受精鸡蛋的预培养}

$0.05 \%$ 高锰酸钾 $\left(\mathrm{KMnO}_{4}\right)$ 擦拭受精鸡蛋, 表面 消毒后, 置于 $37.5^{\circ} \mathrm{C}, 100 \%$ 湿度的孵箱中预培养 $8 \mathrm{~d}$, 待 CAM 的血管网络发育稳定(图 1(a)), 作为发育正 常的 CAM 模型备用。

\section{2 多孔磷酸钙骨修复材料的制备}

CPC 采用 Biocement-D 配方作为固相粉末 ${ }^{[14]}$, 磷酸盐缓冲液(Phosphate buffer solution, PBS) 作为 液相, 按液固比 $1.0 \mathrm{~mL} / \mathrm{g}$ 均匀混合, 得到 CPC 浆体, 将浆体灌入自制模具(一部分含针头，可造通孔，另 一部分不含), 水化后得到 CPC 支架(CPC scaffold, $\mathrm{CPCs}$ ), 其中一半多孔, 一半无孔(图 1(b)中白色圆 柱形试样, 黑色实线为多孔和无孔的分界线), 试样 尺寸 $\phi 8.80 \mathrm{~mm} \times 2.00 \mathrm{~mm}$, 孔径为 $500 \mu \mathrm{m}$ 。多孔 HA 采用 $\mathrm{H}_{2} \mathrm{O}_{2}$ 发泡、 $1250{ }^{\circ} \mathrm{C}$ 烧结而成 ${ }^{[15]}$, 试样尺寸 $\phi 8.80 \mathrm{~mm} \times 2.00 \mathrm{~mm}$, 孔隙率约为 $78 \%$, 孔径为 200 500 $\mu \mathrm{m}$ (图 1(c)白色圆柱形试样)。两种多孔磷 酸钙骨修复材料经超声、清洗、干燥、灭菌后备用。 

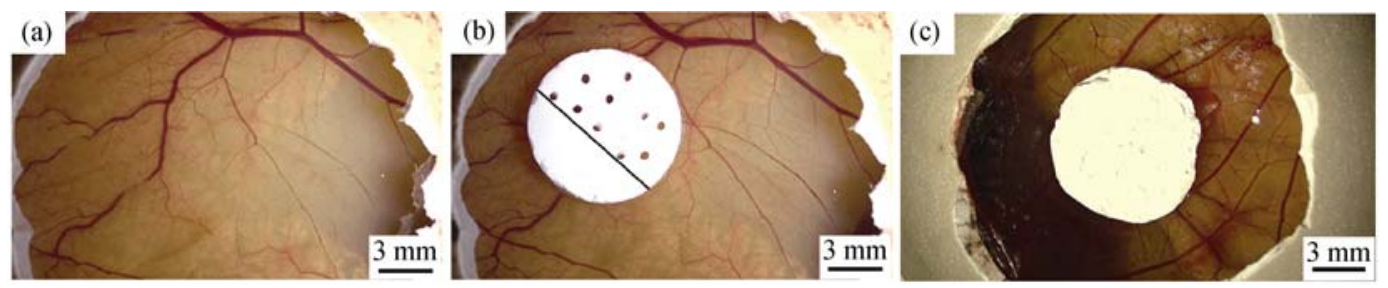

图 1 预培养 $8 \mathrm{~d}$ 的 CAM 血管网络(a), 以及植入 CPCs (b)和 HA (c)后的 CAM

Fig. 1 Vascular network of CAM after pre-incubated for $8 \mathrm{~d}$ (a), CAM after implanted CPCs (b) and HA (c)

\section{3 多孔磷酸钙骨修复材料植入 CAM 模型培养}

取出培养 $8 \mathrm{~d}$ 后的鸡蛋, 用 $75 \%$ 乙醇消毒; 无菌 针头刺破蛋壳顶端; 无菌组织镊掀起蛋壳顶端, 形 成直径约为 $2 \mathrm{~cm}$ 的窗口; 将软壳膜小心揭起并撕去, 暴露 CAM, 将无菌多孔磷酸钻骨修复材料(CPCs 和 HA)分别植入 CAM 中, 如图 1(b)和 1(c)所示。无菌 医用敷贴封口后将鸡蛋置于 $37.5^{\circ} \mathrm{C}, 100 \%$ 湿度的孵 箱(HWS-80, KEWEI, China)继续培养。

\subsection{CPCs 表面血管化定量分析}

培养 2、6 和 $8 \mathrm{~d}$ 后, 采用体式显微镜(CSW-X, COOSWAY, China)观察 CAM 模型中 CPCs 表面的血. 管生长。利用 Image-Pro Plus (IPP)和 Nano Measurer 软件对 CPCs 表面血管生长进行定量统计并分析, 每组 3 个平行样。

\subsection{1 血管密度}

文中血管密度 $d_{\text {blood }}$ 定义为某一特定区域血管 的总面积 $S_{\text {blood }}$ 与该区域总面积 $S_{\text {total }}$ 之比 ${ }^{[12]}$, 如式 (1)。使用 IPP 软件计算 CPCs 多孔和无孔部分以及 整个支架表面的面积，同时计算各区域中血管所占 面积, 利用式(1)分别计算培养 2、6 和 $8 \mathrm{~d}$ 后 CPCs 血管总密度 $d_{\text {total }}, \mathrm{CPCs}$ 中多孔部分血管密度 $d_{\text {porous }}$

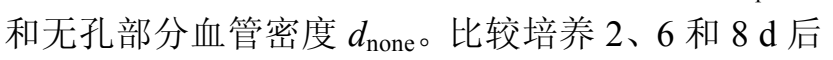
CPCs 中多孔和无孔部分血管密度所占百分比。

$$
d_{\text {blood }}=\frac{S_{\text {blood }}}{S_{\text {total }}}
$$

\subsection{2 血管直径与数量}

利用 Nano Measurer 软件测量培养 2、6 和 $8 \mathrm{~d}$ 后 CPCs 上所有血管的直径, 根据血管直径将其分 为三个等级: $\phi<50 \mu \mathrm{m} 、 50 \mu \mathrm{m}<\phi<100 \mu \mathrm{m}$ 和 $\phi>$ $100 \mu \mathrm{m}^{[16]}$, 比较培养不同时间后不同等级的血管 数量, 以及 $\mathrm{CPCs}$ 中多孔和无孔部分不同等级血管 数量所占的百分比。

\section{5 微观形貌分析}

培养 $8 \mathrm{~d}$ 后, 取出 CAM 模型中的 CPCs, 用 $2.5 \%$ 戊二醛溶液固定 $24 \mathrm{~h}$, 磷酸盐缓冲液 (Phosphate buffer solution, PBS)清洗 3 次, 乙醇梯度 脱水, 临界点干燥, 喷金 ${ }^{[17]}$, 用扫描电子显微镜
(SEM, Scanning electron microscope, FEI-Quanta200, Philips, Netherlands)观察支架表面的血管生长, 利 用 Nano Measurer 软件计算血管直径。

\section{6 组织学表征}

由于磷酸钻材料在 CAM 中培养时间较短, 组 织和血管长入量较少, 脱钻切片困难。因此研究采 用不脱钻硬组织切片技术对材料进行组织学评价。 取出在 CAM 中培养 $4 \mathrm{~d}$ 的 HA, 固定、梯度脱水、 聚甲基丙烯酸甲酯(Polymethyl methacrylate, PMMA) 包埋、切片(SP1600, Leica, Germany)和苏木精-伊红 (Hematoxyin-eosin, H\&E) 染色 ${ }^{[18]}$, 观察长入 HA 的 血管和组织。

\section{7 统计学分析}

实验中所有定量数据均表示为 $\bar{X} \pm S D$, 采用 Student's T test 进行统计学分析: $p<0.05$, 显著性差 异; $p<0.01$, 极显著性差异。

\section{2 结果与讨论}

\subsection{CPCs 表面血管化评价}

图 2 中(a)、(b)和(c)分别为 CPCs 在 CAM 模型 中培养 2、6 和 $8 \mathrm{~d}$ 的体式显微镜照片, 将培养 $8 \mathrm{~d}$ 的 CPCs 表面不同区域 1、2 和 3 放大得到图(d)、(e) 和(f)。从图中可见, 培养初期 $(2 \mathrm{~d}) \mathrm{CPCs}$ 周围有血液 渗出, 表面出现凝血(图 2(a)), 随着培养时间延长, CPCs 表面凝血逐渐消失, 血管数量逐渐增多, 且血 管向 CPCs 内部生长(图 2(b)和(c)), 说明 CPCs 具有 良好的生物相容性, 利于血液循环和凝血消除, 并 促进血管生长。从图 2(a)可见, 培养初期 CAM 组织 及血管没有完全包裹 CPCs，此时 CPCs 表面血管生 长具有随机性，无孔部分血管多于多孔部分(图 2(a) 箭头所示)。随着培养时间延长, CAM 逐渐发育, 其 组织和血管不断生长, 包裹 CPCs, 同时 CPCs 的孔 隙结构为细胞长入和血管循环提供了空间 ${ }^{[19]}$, 因此 CPCs 多孔部分表面血管快速生长, 血管逐渐丰富, 凝血基本消失，而无孔部分因没有足够的空间供细 胞和血管长入，血管生长明显缓慢， CPCs 表面还可 

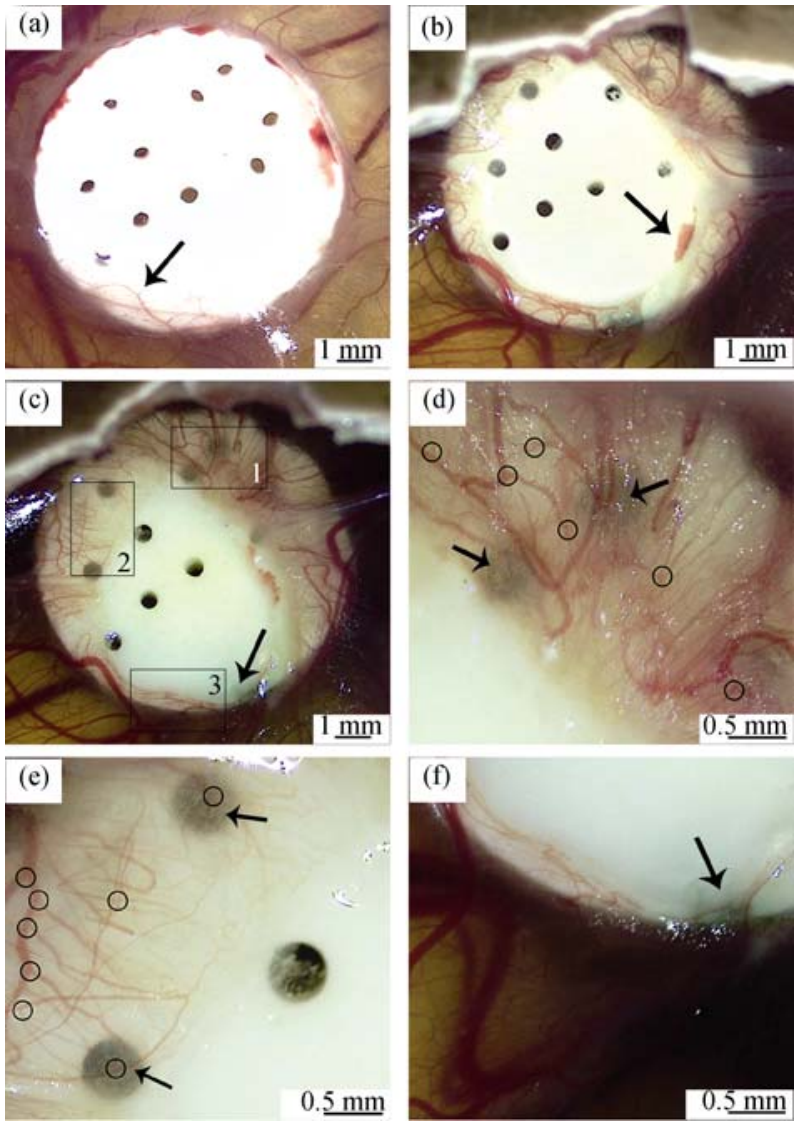

图 $2 \mathrm{CPCs}$ 植入 CAM 模型中培养 $2 \mathrm{~d}(\mathrm{a}) 、 6 \mathrm{~d}(\mathrm{~b})$ 和 $8 \mathrm{~d}(\mathrm{c})$ 后其 表面的血管生长，以及培养 $8 \mathrm{~d}$ 后放大 CPCs 表面不同区域(1 (d)、2 (e)和 3 (f))的血管生长的体式显微镜照片

Fig. 2 Angiogensis on the surface of CPCs after implanted in CAM model for $2 \mathrm{~d}(\mathrm{a}), 6 \mathrm{~d}$ (b) and $8 \mathrm{~d}$ (c). Angiogensis of different parts (1 (d), 2 (e) and 3 (f)) on the surface of CPCs after implanted for $8 \mathrm{~d}$

见大块凝血(图 2(b)箭头所示), 到第 $8 \mathrm{~d}$ 无孔部分还 出现血管退化现象(图 2(c)箭头所示)。由图 2(d)和图 2(e)可见, 在 CPCs 多孔部分, 血管以出芽方式生长, 形成血管网, 符合血管正常生理结构 ${ }^{[20]}$ (图 2(d) 和 2(e)中圆圈所示为血管分支点), 同时血管向孔隙的 中心汇聚(图 2(d)和 2(e)中箭头所示); 图 2(f)中无孔 部分表面血管沿支架轮廓方向生长，血管分支点较 少, 且血管数量少, CPCs 表面有血栓形成(图 2(f)箭 头所示)。结果表明 CPCs 表面血管生长初期具有随 机性，而孔隙结构对后期血管生长以及血管网的形 成起明显的促进作用, 并可以引导血管的生长方向。 但是本实验中 CPCs 孔隙尺寸和结构与正常骨的尚 有一定差距, 因此实验还需要进一步优化。

\subsection{1 血管密度}

图 3(a) 为在 CAM 模型中培养 2、6 和 $8 \mathrm{~d}$ 后 CPCs 表面血管总密度, 图 3(b)为多孔和无孔部分血管密 度所占百分比。由图 3(a)可见，随着培养时间增加，
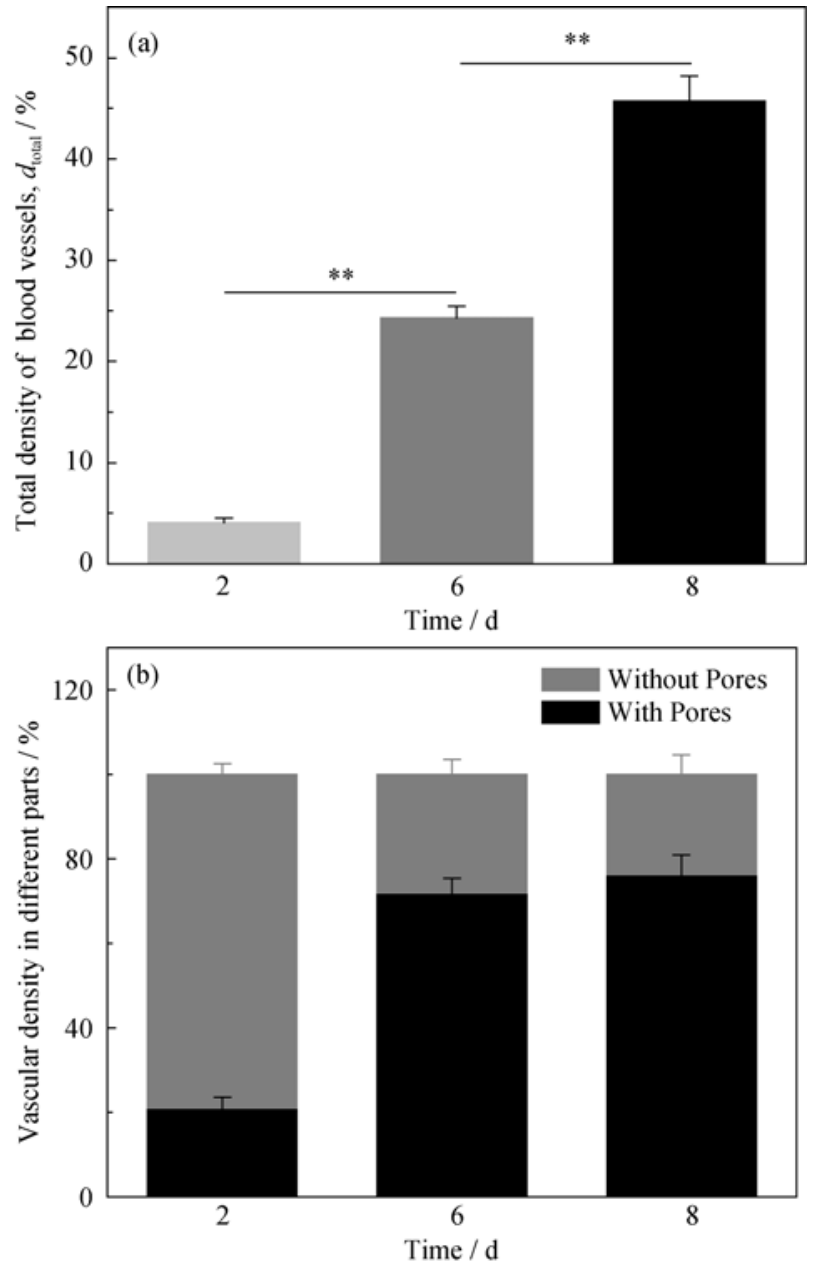

图 3 培养 $2 、 6$ 和 $8 \mathrm{~d}$ 后 CPCs 表面血管总密度(a), 以及 CPCs 表面多孔和无孔部分血管密度所占百分比(b)

Fig. 3 Total density of blood vessels on CPCs after implanted in CAM model for 2, 6 and $8 \mathrm{~d}$ (a). The percentage of vascular densities of CPCs, with pores and without pores (b)

$* * p<0.01, n=3$

CPCs 表面血管总密度极显著增加, 培养 $2 \mathrm{~d}$ 后血管 总密度仅为 $(4.08 \pm 0.42) \%$, 培养 $8 \mathrm{~d}$ 后血管总密度 可达(45.73 \pm 2.46$) \%$ 。由图 3(b)可见，培养 $2 \mathrm{~d}$ 后， $\mathrm{CPCs}$ 无孔部分血管密度大于多孔部分, 这是由于 培养初期 CAM 没有完全包裹 CPCs, 其表面血管生 长具有随机性。随着培养时间增加, 孔隙结构对于 血管生长的优势逐渐明显，血管逐渐向多孔部分表 面生长, 无孔部分表面血管生长受到限制, 因此多 孔部分血管密度逐渐增加, 所占比例超过无孔部 分。图 3 的定量数据说明了多孔结构对骨修复材料 血管化的重要性。

\subsection{2 血管直径与数量}

图 4 为在 CAM 中培养 $2 、 6$ 和 $8 \mathrm{~d}$ 后, CPCs 表 面血管的直径分布(图 4(a))、不同直径的血管总数 (图 4(b))和 CPCs 表面多孔部分和无孔部分不同血管 直径数量所占百分比(图 4(c))。由图 4(a) 和 4(b) 可见, 
培养初期, CPCs 表面血管直径 $(\phi)$ 较小，基本在 $100 \mu \mathrm{m}$ 内。随着培养时间增加, 出现 $\phi>100 \mu \mathrm{m}$ 的血. 管, 同时 $\phi<50 \mu \mathrm{m}$ 的血管数量显著增加 $(6 \mathrm{~d})$, 培养 $8 \mathrm{~d}$
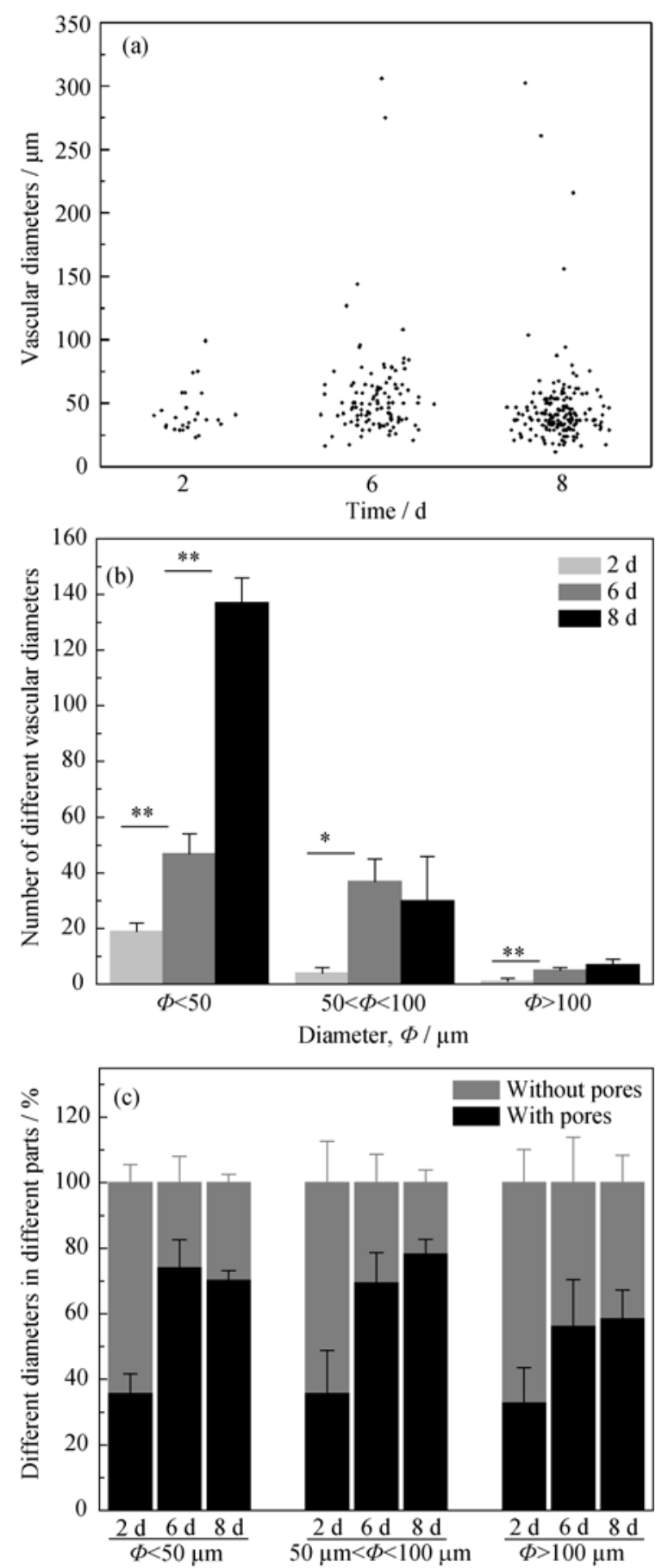

图 4 培养 $2 、 6$ 和 $8 \mathrm{~d}$ 后 $\mathrm{CPCs}$ 表面血管的直径分布(a), CPCs 表面不同直径血管 $(\Phi<50 \mu \mathrm{m}, 50 \mu \mathrm{m}<\Phi<100 \mu \mathrm{m}$ 和 $\Phi>100 \mu \mathrm{m})$ 总数量(b), 以及 $\mathrm{CPCs}$ 表面多孔和无孔部分中不同直径血管 的数量所占百分比(c)

Fig. 4 The distribution of vascular diameters (a), numbers of different vascular diameters $(\Phi<50 \mu \mathrm{m}, 50 \mu \mathrm{m}<\Phi<100 \mu \mathrm{m}$ and $\Phi>100 \mu \mathrm{m})(\mathrm{b})$ and the percentage of different diameters in different parts (with pores and without pores) (c) on the surface of CPCs after implanted for 2, 6 and $8 \mathrm{~d}$ ${ }^{*} p<0.05, * * p<0.01, n=3$
后 $\phi<50 \mu \mathrm{m}$ 的血管仍显著性增加，而 $50 \mu \mathrm{m}<\phi<100 \mu \mathrm{m}$ 和 $\phi>100 \mu \mathrm{m}$ 的血管生长缓慢, 数量无明显增加。结 合图 2(b)和 2(c)可知, CPCs 表面血管生长主要有两种 形式: 一种为覆盖在 CPCs 表面, 随着 CAM 发育不 断生长的血管, 其直径较大; 一种为 $\mathrm{CPCs}$ 上大血管 按出芽方式生长形成的血管, 其直径较小。CPCs 上 $\phi>50 \mu \mathrm{m}$ 的血管多为第一种生长方式, $\phi<50 \mu \mathrm{m}$ 的血 管多为第二种方式，当 CAM 中大血管生长覆盖 $\mathrm{CPCs}$ 表面后，以细小血管 $(\phi<50 \mu \mathrm{m})$ 生长为主。图 4(c)与图 3(b)结果相似, 表明孔隙结构利于血管包 绕、穿过，可以促进血管生长。

\section{2 微观形貌分析}

图 5 为 CPCs 植入 CAM $8 \mathrm{~d}$ 后表面的 SEM 照片, 从图 5(a)可以明显观察到支架表面存在呈出芽方式 生长的血管，其平均直径约为 $20 \mu \mathrm{m}$, 将支架表面 上的血管进一步放大得到图 5(b), 图中可见 CAM 的膜结构将血管包裹，且膜结构贴覆于 CPCs，可以 推测血管紧贴 CPCs 生长。

\section{3 组织学表征}

图 6 是 CAM 模型中培养 $4 \mathrm{~d}$ 后 HA 的 H\&E 染 色图。图 6(a)和图 6(b)均可见 HA 边缘被大量疏松 结缔组织包围, HA 孔隙中存在散落的红细胞(图 6(a) 黑色箭头所示), 这可能是在切片过程中, 长入 HA 内部的血管受损破裂，血管内部的红细胞散落于孔
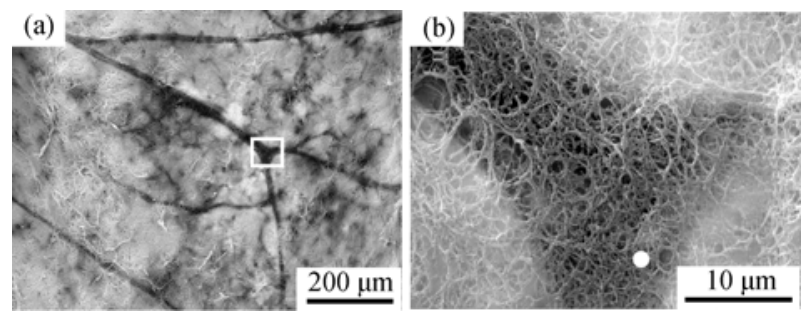

图 5 植入 CAM $8 \mathrm{~d}$ 后 CPCs 表面的 SEM 照片

Fig. 5 SEM images of the surface on CPCs after implanted in CAM model for $8 \mathrm{~d}$
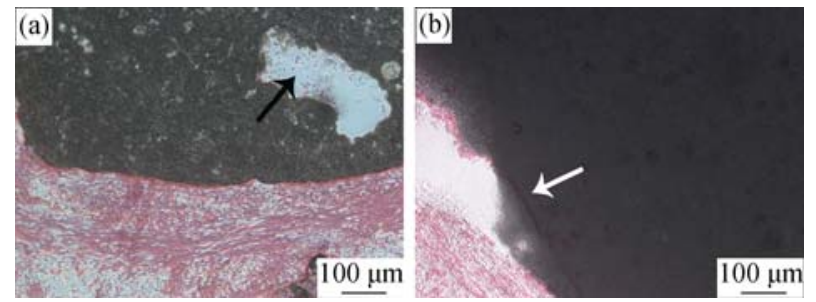

图 6 多孔羟基磷灰石陶瓷植入 CAM $4 \mathrm{~d}$ 后不同部位的 H\&E 染色

Fig. 6 H\&E staining images of porous hydroxyapatite ceramic after implanted for $4 \mathrm{~d}$ in CAM model

Black arrow: red blood cell grew into the inner pore of HA, white arrow: blood vessel grew into the inner pore of HA 
隙中。图 6(b)可见细管状物质随疏松结缔组织延伸, 长入 HA 内部, 并分支呈网状结构(图 6(b)白色箭头 所示)。H\&E 染色结果说明血管通过出芽方式生长 进入 HA 内部形成血管网, 表明孔隙结构利于细胞 和血管向材料内部生长，促进材料血管化，这与 CPCs 血管化定量分析结果一致。但由于组织学制片 技术的局限, 目前在 CAM 模型中培养后的 CPCs 试 样的组织学切片正在探索中。

\section{3 结论}

利用具有丰富血管的 CAM 建立半体内模型, 研究多孔磷酸钻骨修复材料的血管化。CPCs 表面血 管生长的结果表明, 在培养初期, CAM 的组织和血. 管未完全包裹 CPCs, CPCs 表面血管生长具有随机 性; 在培养后期，由于孔隙结构利于血管包绕、穿过, 对 CPCs 表面血管生长有明显的促进作用, 同时孔 隙结构利于血管网的形成和维持。CAM 模型中培养 HA 的组织学结果表明孔隙结构利于细胞和血管长 入, 对材料的血管化非常重要。本研究建立的 CAM 模型，较动物植入实验更简便快捷，解决了动物实 验操作困难、影响因素复杂、实验周期长等缺点, 为 快速简便地评价植入材料的血管化提供了新方法, 具有较好的应用前景。

\section{参考文献:}

[1] KARAGEORGIOU V, KAPLAN D. Porosity of 3D biomaterial scaffolds and osteogenesis. Biomaterials, 2005, 26(27): 5474-5491.

[2] VAHABZADEH S, ROY M, BOSE S. Effects of silicon on osteoclast cell mediated degradation, in vivo osteogenesis and vasculogenesis of brushite cement. Journal of Materials Chemistry B, 2015, 3(46): 8973-8982.

[3] ZHANG W J, CHANG Q, XU L, et al. Graphene oxide-copper nanocomposite-coated porous $\mathrm{CaP}$ scaffold of vascularized bone regeneration via activation of Hif-1 $\alpha$. Advanced Healthcare Materials, 2016, 5(11): 1299-1309.

[4] NANDI S K, GHOSH S K, KUNDU B, et al. Evaluation of new porous $\beta$-tri-calcium phosphate ceramic as bone substitute in goat model. Small Ruminant Research, 2008, 75(2/3): 144-153.

[5] DERYUGINA E I, QUIGLEY J P. Chick embryo chorioallantoic membrane models to quantify angiogenesis induced by inflammatory and tumor cells or purified effector molecules. Methods in Enzymology, 2008, 444: 21-41.

[6] LI M, PATHAK R R, LOPEZ-RIVERA E, et al. The in ovo chick chorioallantoic membrane (CAM) assay as an efficient xenograft model of hepatocellular carcinoma. Journal of Visualized Experiments, 2015(104): e52411.

[7] PATIL R S, SHAH S U, SHRIKHANDE S V, et al. IL17 producing $\gamma \delta \mathrm{T}$ cells induce angiogenesis and are associated with poor survival in gallbladder cancer patients. International Journal of Cancer, 2016, 139(4): 869-881.

[8] PORCÙ E, PERSANO L, RONCA R, et al. The novel antitubulin agent TR-764 strongly reduces tumor vasculature and inhibits Hif-1 $\alpha$ activation. Scientific Reports, 2016, 6: 27886.

[9] CHIEN S Y, HUANG C Y, TSAI C H, et al. Interleukin-1 $\beta$ induces fibroblast growth factor 2 expression and subsequently promotes endothelial progenitor cell angiogenesis in chondrocytes. Clinical Science, 2016, 130(9): 667-681.

[10] PRICE C F, BURGESS D J, KASTELLORIZIOS M. L-DOPA as a small molecule surrogate to promote angiogenesis and prevent dexamethasone-induced ischemia. Journal of Controlled Release, 2016, 235: 176-181.

[11] SCHEEL J, HERMANN M. Integrated risk assessment of a hydroxyapatite-protein-composite for use in oral care products: a weight-of-evidence case study. Regulatory Toxicology and Pharmacology, 2011, 59(2): 310-323.

[12] MAGNAUDEIX A, USSEGLIO J, LASGORCEIX M, et al. Quantitative analysis of vascular colonisation and angio-conduction in porous silicon-substituted hydroxyapatite with various pore shapes in a chick chorioallantoic membrane (CAM) model. Acta Biomaterialia, 2016, 38: 179-189.

[13] BUSCHMANN J, HÄRTER L, GAO S P, et al. Tissue engineered bone grafts based on biomimetic nanocomposite PLGA/amorphous calcium phosphate scaffold and human adipose-derived stem cells. Injury, 2012, 43(10): 1689-1697.

[14] HUANG P, LI P, ZHAO J S, et al. Mechanical activation reinforced porous calcium phosphate cement. Journal of Inorganic Materials, 2015, 30(4): 432-438.

[15] ZHANG H J, ZHU X D, WANG X L, et al. Effect of sintering processes on surface properties and protein adsorption of hydroxyapatite ceramic particles. Journal of Inorganic Materials, 2010, 25(7): 770-774.

[16] JU L H, HU P, NI S. Quantitative analysis of blood vessels in chick embryo chorioallantoic membranes through digital imaging. Journal of Clinical Rehabilitative Tissue Engineering Research, 2008, 12(13): 2463-2466.

[17] VALDES T I, KREUTZER D, MOUSSY F. The chick chorioallantoic membrane as a novel in vivo model for the testing of biomaterials. Journal of Biomedical Materials Research Part A, 2002, 62(2): 273-282.

[18] DACULSI G, PASSUTI N, MARTIN S, et al. Macroporous calcium phosphate ceramic for long bone surgery in humans and dogs. Clinical and histological study. Journal of Biomedical Materials Research Part A, 1990, 24(3): 379-396.

[19] BOSE S, ROY M, BANDYOPADHYAY A. Recent advances in bone tissue engineering scaffolds. Trends in Biotechnology, 2012, 30(10): $546-554$.

[20] MASTERS B R. Fractal analysis of the vascular tree in the human retina. Annual Review of Biomedical Engineering, 2004, 6: 427-452. 